\title{
Intuitive control of a prosthetic elbow
}

\author{
Manelle Merad, Étienne de Montalivet, Agnès Roby-Brami, and Nathanaël Jarrassé
}

\begin{abstract}
Many transhumeral amputees deplore the lack of functionality of their prosthesis, mostly caused by a counterintuitive control strategy. This work is the first implementation of an automatic prosthesis control approach based on natural coordinations between upper limb joints and IMU-based humeral orientation measurement. One healthy individual was able to use the prosthetic elbow, fitted with a prosthetic forearm and attached to the subject's upper arm, to point at targets with an encouragingly small error.
\end{abstract}

\section{INTRODUCTION}

Transhumeral amputations impair severely a person's ability to perform activities of daily living (ADLs). The missing limb replacement by a prosthetic device is then often required. Among commercialized solutions, there are passive prosthetic elbows (12K44 ErgoArm $®$ Hybrid Plus, Ottobock(C) that can be locked manually into a desired position, as well as active prosthetic elbows (DynamicArm 12K100, Ottobock(C), and UtahArm3+, Motion Control, Inc.), that are combined with myoelectric wrists and hands (mainly VarySpeed hand, Ottobock(c)). The most common strategy to control an upper limb prosthetic limb is myoelectric control which uses the residual muscles contractions to initiate the prosthesis motion. Transhumeral amputees control the hand, wrist, and sometimes even elbow motion with only two control signals (usually biceps and triceps when available). Myoelectric control requires the user to decompose prosthetic movements, and to focus intensively on the prosthesis control and motion. Many research groups are seeking solutions to improve myoelectric control [1], but alternatives to myoelectric signals as control inputs [2] are also investigated.

Contrary to prosthetic movements, natural human upper limb movements are intuitive and object-centered: instead of focusing on the movement of each joint along the upper limb, one concentrates on the task to achieve. This ability is explained by the existence of a coordination between the joint kinematics [3]-[5]. This concept when applied to prosthetics yields the automatized motion of replaced joints, like a prosthetic elbow, based on an inter-joint coordination model relating residual and missing joints kinematics. Several studies have shown that distal joint motion could be predicted from proximal joints kinematics using a model of coordination between upper limb joints [6], [7]; however, residual limb motion were measured with motion capture

The authors are with CNRS, UMR 7222, ISIR, F-75005 Paris, France, also with INSERM, U1150, Agathe-ISIR, F-75005 Paris, France, and also with Sorbonne Universit'es, UPMC Univ Paris 06, UMR 7222, ISIR, F-75005 Paris, France, \{merad, roby-brami, jarrasse\}@isir.upmc.fr systems that were not compatible with outside-the-lab environments, and thus, the control laws were not implemented on real prostheses.

An automatic prosthetic control method, based on natural coordinations between shoulder and elbow kinematics, was implemented, and this paper presents the obtained results. An healthy individual was able to point at targets with an automatically-driven prosthetic forearm (1 degree of freedom (DoF)). Inertial measurement unit (IMU)-based arm orientation were used as input signals of the prosthesis controller.

\section{MATERIALS AND METHODS}

One right-handed subject (male, 24 years old, $182 \mathrm{~cm}$ ) participated in the study. Two consecutive parts, the training data set acquisition and the control test, constituted the experimental session. In the first part, the subject performed pointing movements while the arm and forearm kinematics were recorded. The inter-joint coordination model was built from the training data set, and drove the prosthesis during the second part of the experiment.

Experimental setup: The prosthesis was designed based on the functional characteristics of commercialized active elbow prostheses. The prototype, shown in Fig. 1A, was controlled by a Raspberry $\mathrm{Pi}$, which controlled the motor driver and read the data from an x-IMU (x-io Technologies, Ltd.) (Fig. 1A). The subject used a rod attached to the back of a wrist splint or to the prosthesis in order to reach the targets. The targets were represented by a bright push button mounted on a $7-\mathrm{DoF}$ robotic arm $\left(\mathrm{WAM}^{\mathrm{TM}}\right.$ arm, Barrett Technology, Inc.). The prosthesis controller processed only IMU signals, however, for analysis purpose, upper limb motion was recorded with a camera-based motion capture system, Codamotion (Charnwood Dynamics, Ltd.). Seven markers were placed on the subject's arm, and the eighth measured the prosthesis rod tip position (Fig. 1A). The experimental session was videotaped.

Experimental protocol: During the first part of the experiment, the subject pointed at 19 targets in front of him (workspace dimension: 20x60x60 $\mathrm{cm}^{3}$ ), with the rod attached to the wrist splint. Upper limb kinematics are influenced by external weights applied on the arm segments, thus the prosthesis was attached to the subject, in an inactive mode (locked into an extended position), even during the training session. The starting position was defined with the subject's forearm on the arm rest (Fig. 1A). For each target, the subject stayed for $2 \mathrm{~s}$ at the starting position, went towards the target, pushed the button with the rod tip, stayed immobile for $2 \mathrm{~s}$, and went back to the starting position, while the WAM robotic arm's end effector was moving to the next 

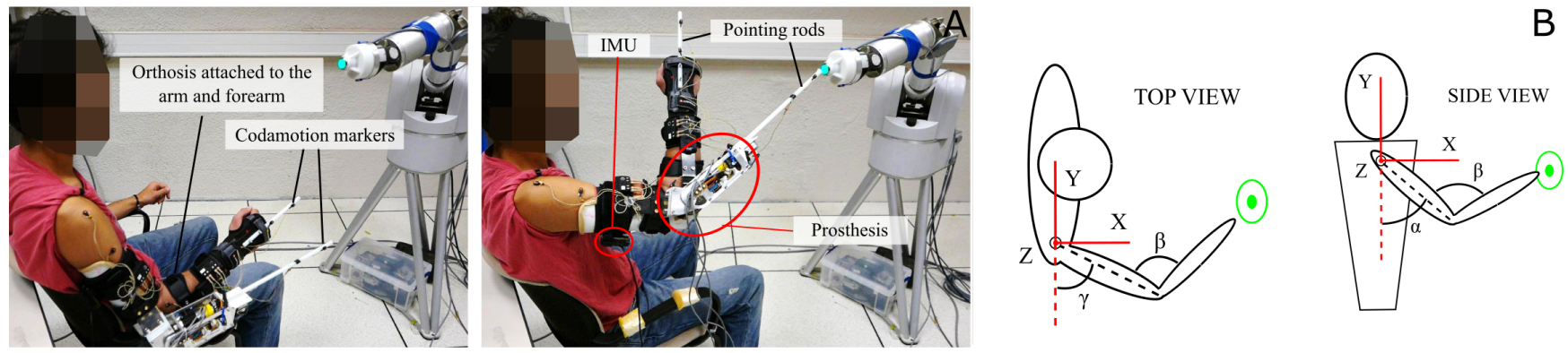

Fig. 1. The elbow prosthesis, to which an IMU is connected, was mounted on an orthosis attached to the participant's arm and forearm. Equipped with Codamotion markers, the subject is pointing at targets with a rod attached to a wrist splint during the training data acquisition or to the prosthetic elbow during the control test. Targets were presented by a WAM robotic arm. B: Anatomical angles $\gamma, \alpha, \beta$ describe the upper limb posture.

target location. One trial consisted in pointing at 19 targets, and was repeated three times. Offline, the shoulder angles, $\gamma$ the humeral direction and $\alpha$ the humeral inclination, were derived from the $\mathrm{x}$-IMU data, while the elbow angle, $\beta$, was derived from Codamotion data (Fig. 1B). A Radial Basis Function Network (RBFN)-based regression was performed to model the inter-joint relationship between $(\dot{\gamma}, \dot{\alpha})$ and $\dot{\beta}$. Kinematic data from 10 targets were included in the training data set; including more targets would result in over-training effects. During the second part of the experiment, the IMU was connected to the prosthesis controller that ran the embedded RBFN-based regression algorithm with the model parameters obtained during the training phase. The subject's arm was locked into a constant position with the elbow orthosis. The subject pointed at the same targets as previously using a rod that extended the prosthetic forearm (Fig. 1A).

\section{RESULTS}

The task performance was assessed with the precision error and the movement duration. The precision error between the position reached by the prosthesis end tool and the target was $1.5 \mathrm{~cm} \pm 1.2 \mathrm{~cm}$ averaged over all targets. The time needed to reach the target naturally, i.e. with the rod attached to the hand, is $1.56 \mathrm{~s} \pm 0.20 \mathrm{~s}$, while the time needed to reach the target with the rod attached to the prosthesis is $2.33 \mathrm{~s} \pm$ $0.42 \mathrm{~s}$. The results are depicted in Fig. 2.

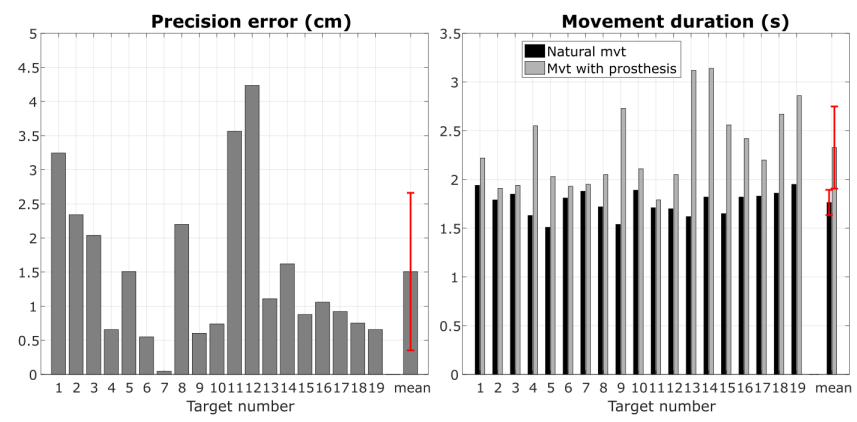

Fig. 2. The pointing movements are assessed with the precision error (distance between rod tip and target) and the movement duration (time needed to reach the target), which is compared to the natural movement duration from the first training trial. Values are averaged over all targets; mean and standard deviation values are displayed.

\section{Discussion}

One healthy subject was able to reach targets using a prosthetic elbow, driven by his own inter-joint coordination model. To the authors' knowledge, this study is the first reported implementation of such control strategy using IMUbased control input signals. Although the algorithm was trained on 10 targets and tested on 19, the low precision error demonstrates the control strategy's spatial generalization property. The fact that the subject slowed down his movements when using the prosthesis could suggest the emergence of compensatory strategies. They will be investigated in further analyses.

\section{CONCLUSiON}

Based on natural inter-joint coordinations, the control strategy developed in this paper is intuitive. The subject did not learn how to control the prosthesis prior to the experimental session: the reported results correspond to his first and only trial. Thus, better results could be expected with short training. Learning and control training effects will be further investigated. We will also work on the development of a inter-joint coordination generic model for future tests with amputated patients.

\section{REFERENCES}

[1] C. Castellini, P. Artemiadis, M. Wininger, A. Ajoudani, M. Alimusaj, A. Bicchi, B. Caputo, W. Craelius, S. Dosen, K. Englehart, et al., "Proceedings of the first workshop on peripheral machine interfaces: going beyond traditional surface electromyography," Front. Neurorobot., vol. 8, 2014.

[2] J. Lobo-Prat, P. N. Kooren, A. H. Stienen, J. L. Herder, B. F. Koopman, and P. H. Veltink, "Non-invasive control interfaces for intention detection in active movement-assistive devices," J. Neuroeng. Rehabil., vol. 11, no. 1, p. 1, 2014.

[3] J. Soechting and F. Lacquaniti, "Invariant characteristics of a pointing movement in man," J. Neurosci., vol. 1, no. 7, pp. 710-720, 1981.

[4] F. Lacquaniti and J. F. Soechting, "Coordination of arm and wrist motion during a reaching task," J. Neurosci., vol. 2, no. 4, pp. 399408, 1982.

[5] A. Roby-Brami, N. Bennis, M. Mokhtari, and P. Baraduc, "Hand orientation for grasping depends on the direction of the reaching movement," Brain Res., vol. 869, no. 1, pp. 121-129, 2000.

[6] M. Popović and D. Popović, "Cloning biological synergies improves control of elbow neuroprostheses," IEEE Eng. Med. Biol. Mag., vol. 20, no. 1, pp. 74-81, 2001

[7] R. R. Kaliki, R. Davoodi, and G. E. Loeb, "Evaluation of a noninvasive command scheme for upper-limb prostheses in a virtual reality reach and grasp task," IEEE Trans. Biomed. Eng., vol. 60, no. 3, pp. 792-802, 2013. 